\title{
DISTRIBUCIÓN POR EDADES DEL MELANOMA DE ÚVEA Y SU RELACIÓN CON LA SUPERVIVENCIA
}

\section{AGE DISTRIBUTION OF UVEAL MELANOMA AND ITS RELATIONSHIP TO SURVIVAL}

\author{
GRAELL X ${ }^{1}$, CAMINAL JM ${ }^{2}$, MASUET C ${ }^{3}$, ARIAS L ${ }^{1}$, RUBIO Mํ․ PUJOL O ${ }^{1}$, ROCA G $^{1}$, \\ ARRUGA J ${ }^{2}$
}

\section{RESUMEN}

Propósito: Estudiar la distribución por edades del melanoma de úvea y relacionarlo con la supervivencia

Método: Se ha realizado un estudio retrospectivo en pacientes diagnosticados de melanoma de úvea. Se han analizado entre otras las variables clínicas: edad, sexo, origen y tamaño tumoral, tiempo de seguimiento, estado sistémico actual, fecha y causa de muerte. Resultados: Se han estudiado 303 pacientes afectos de melanoma de úvea. La edad media de los pacientes fue de 60,09 años. La supervivencia en los pacientes < 50 años a los 2,5 y 10 años de seguimiento es del $91,41 \%, 81,83 \%$ y $61,45 \%$ respectivamente. La supervivencia en los pacientes $\geq 50$ años a los 2, 5 y 10 años es del 90,86\%, 73,18\% y $58,28 \%$ respectivamente, diferencia no estadísticamente significativa entre estos dos grupos de edad. Cuando consideramos el factor sexo en los pacientes $\geq 50$ años encontramos una mayor supervivencia entre los hombres respecto a las mujeres (logrank; $\mathrm{p}=0,038$ ).

Conclusiones: El melanoma de úvea en nuestro medio sigue un patrón de distribución por edades
ABSTRACT

Purpose: To study the age distribution and survival in patients with uveal melanoma.

Methods: A retrospective study was performed on 303 patients diagnosed with uveal melanoma. We analysed the clinical characteristics: age, gender, tumor size and origin, follow-up time, systemic state, survival time and cause of death.

Results: The median age of the patients was 60.09 years. The 2-, 5-, and 10-year survival of patients less than 50 years of age at diagnosis was $91.41 \%$, $81.83 \%$ and $61.45 \%$ respectively. The $2-, 5-$ and $10-$ year survival of patients equal to or older than 50 years was $90.86 \%, 73.18 \%$ and $58.28 \%$ respectively. No significant difference was found between these two age groups. When we considered a possible relationship between the sex factor and survival, in patients equal to or older than 50 years of age, we found a higher survival in men than in women (logrank test; $\mathrm{p}=0.038$ ).

Conclusions: Uveal melanoma in Spain has a similar age distribution to that of other countries, and it is not an infrequent diagnosis in patients under 40 years of age. Survival rates are also similar to that

\footnotetext{
Recibido: 31/5/06. Aceptado: 18/4/07.

Hospital Universitari de Bellvitge. L'Hospitalet de Llobregat. Barcelona. España.

1 Licenciado en Medicina. Servicio de Oftalmología.

2 Doctor en Medicina. Servicio de Oftalmología.

3 Licenciada en Medicina. Servicio de Medicina Preventiva.

Correspondencia:

Xavier Graell

Hospital Universitari de Bellvitge

C/. Feixa Llarga, s/n

08907 L'Hospitalet de Llobregat (Barcelona)

España

E-mail:xgraell@csub.scs.es
} 
muy similar al descrito en otras series de otros países, no siendo infrecuente el diagnóstico en pacientes jóvenes $<40$ años. Las tasas de supervivencia también son similares a otras series. No se han encontrado diferencias significativas entre la edad de los pacientes y el pronóstico vital, aunque analizando subgrupos vemos que los hombres $\geq 50$ años presentan una mejor supervivencia que las mujeres $\geq 50$ años.

Palabras clave: Melanoma de úvea, distribución por edad, tasa de supervivencia, pacientes jóvenes, factor sexo. of other series. We have not found any significant difference between the age of our patients and the survival, although if we analysed the subgroups, we found that the men equal to or over 50 years of age had a better survival than the women of the same age (Arch Soc Esp Oftalmol 2007; 82: 343-348).

Key words: Uveal melanoma, age distribution, survival rates, young patients, sex factor.

\section{INTRODUCCIÓN}

El melanoma de úvea es la tumoración maligna intraocular primaria más frecuente del adulto. $\mathrm{Su}$ incidencia en la población general es bastante baja presentándose solo de 6-7,4\% casos por millón de población por año $(1,2)$.

Aproximadamente el $80 \%$ de los melanomas uveales son de coroides, el $12 \%$ son del cuerpo ciliar y un $8 \%$ son de iris. La edad media de aparición son los 55 años y es raro en jóvenes y niños $(1,3)$. En nuestro medio es más frecuente la aparición en gente de etnia caucásica y existe cierta predisposición para los ojos claros (1).

Muchos aspectos como la epidemiología, la patogénesis o el tratamiento de esta neoplasia continúan siendo controvertidos. Hemos realizado este estudio para investigar la epidemiología de esta neoplasia, en concreto queríamos conocer cómo se distribuye el melanoma de úvea según la edad y ver si existe alguna relación entre este factor epidemiológico y la supervivencia de estos pacientes, puesto que no existen trabajos publicados en España al respecto.

\section{SUJETOS, MATERIAL Y MÉTODO}

Realizamos un estudio retrospectivamente de serie de casos clínicos de pacientes diagnosticados de melanoma uveal entre los años 1984 y 2005 de nuestra base de datos, en la sección de Oncología Ocular de nuestro centro. Se incluyen tanto a los pacientes de las poblaciones del área de influencia de nuestro hospital como a los que nos fueron remitidos de otras zonas de España.
Analizamos las variables clínicas: edad, sexo, ojo afecto, origen y tamaño tumoral, clasificación según el COMS (Collaborative Ocular Melanoma Study), fecha de último control, tiempo de seguimiento, estado vital (fallecimiento atribuible al tumor o no atribuible, o vivo), fecha y causa de fallecimiento.

Los datos fueron analizados con el paquete informático SPSS 11.0, siendo comparados los datos categóricos con el test Chi-cuadrado, o Fisher según combino y fue estudiada la supervivencia por medio de las curvas de Kaplan-Meier y comparándolas con la prueba log-Rank.

\section{RESULTADOS}

Se estudiaron 303 pacientes afectos de melanoma de úvea diagnosticados en nuestro servicio, siendo el $44,2 \%$ hombres (n: 134$)$ y el $55,8 \%$ mujeres (n: 196). La edad media de los pacientes fue de 60,09 años (IC 95\%, 58,42-61,77). El ojo derecho fue afectado en el $52,7 \%$ (n: 160) y el izquierdo en el 47,3\% (n: 143).

El $84,5 \%$ de pacientes presentaban una tumoración coroidea (n: 256), el 13,2\% una tumoración en el cuerpo ciliar (n: 40) y un $2,3 \%$ en el iris (n: 7). En cuanto al tamaño de las tumoraciones, la altura media fue de 6,2 (IC 95\%, 5,8-6,6), y el diámetro basal máximo de 11,9 (IC 95\%, 11,3-12,5).

Según el COMS en nuestra serie encontramos un 9,9\% de pacientes en el grupo COMS 1 (n: 30), un $61,4 \%$ en el COMS 2 (n: 186), y un $28,7 \%$ en el COMS 3 (n: 87).

El tiempo medio de seguimiento fue de 1144,6 días (IC 95\%, 1041-1248,2). 
De los 303 pacientes, $253(83,8 \%)$ se encontraban vivos y con buen estado sistémico; 22 (7,3\%) estaban vivos pero con presencia de metástasis atribuibles al melanoma; $27(8,9 \%)$ estaban muertos; solo en un paciente $(0,3 \%)$ no se pudo conocer su estado vital (fig. 1).

La distribución por edades del melanoma de úvea de nuestra serie se refleja en la figura 2, en la que se aprecia una distribución bimodal de frecuencia de melanoma en los grupos de edad de diagnóstico comprendida entre los 51-60 años y entre los 66-75 años.

Fue especialmente poco frecuente entre aquellos pacientes diagnosticados de un melanoma coroideo antes de los $20(1 ; 0,3 \%), 30(6 ; 2,0 \%)$ y 40 años $(38 ; 12,5 \%)$.

No se han encontrado diferencias estadísticamente significativas entre la localización anatómica de los tumores, y las diferentes edades de los pacientes ( $>>0,05)$, así en los pacientes de menos de 50 años el $97,1 \%$ de los tumores son de localización coroidea, y en los pacientes de 50 o más años lo son el $97,9 \%(\mathrm{p}=0,656)$.

Tampoco hemos encontrado diferencias estadísticamente significativas $(p>0,05)$ entre el tamaño tumoral y los diferentes grupos de edades. En los pacientes de menos de 50 años el 79,3\% presenta-

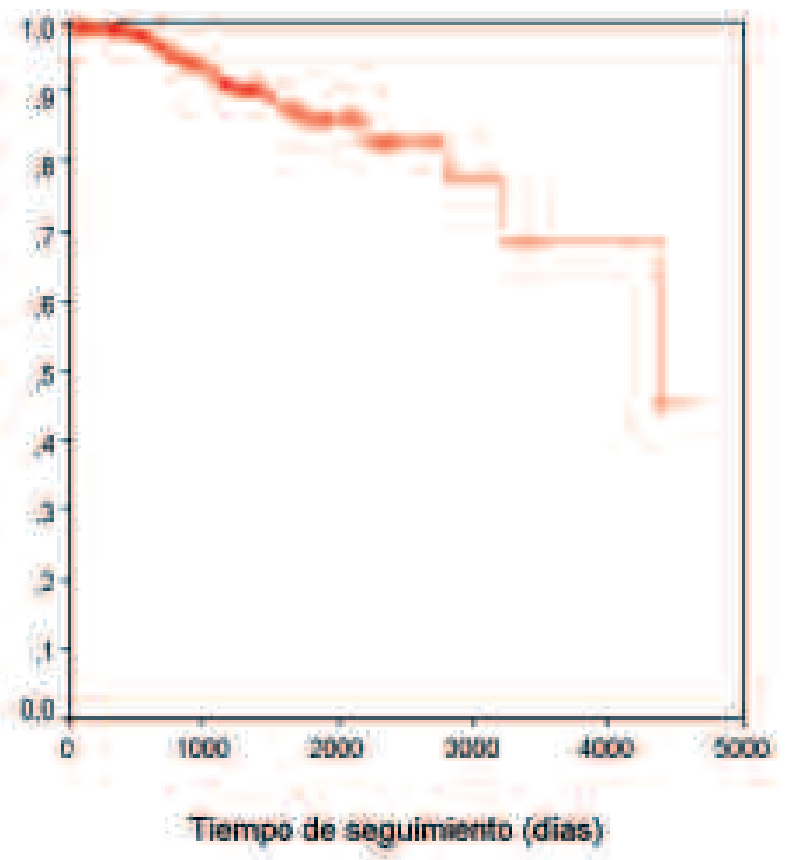

Fig. 1: Curva de supervivencia de Kaplan-Meier de nuestra serie de pacientes con melanoma de úvea.

\section{Edad}

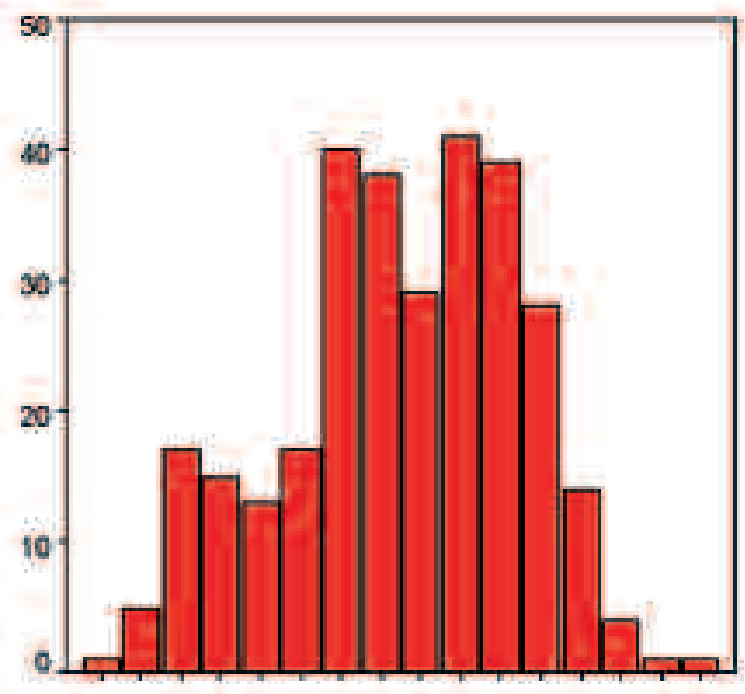

Edad

Fig. 2: Distribución por edades del melanoma de úvea.

ron una tumoración pequeña o mediana (COMS 1 o 2) y el $20,7 \%$ un tumor grande (COMS 3), en los pacientes de más de 50 años esta proporción fue del $69,8 \%$ para el primer grupo y de $30,2 \%$ en el segundo $(\mathrm{p}=0,154)$.

Cuando analizamos la edad de los pacientes (menos de 50 años respecto a los de 50 o más años) en relación a la supervivencia, no hemos encontrado diferencias estadísticamente significativas ( $\mathrm{p}=0,323$ ) (fig. 3). La supervivencia en los pacien-

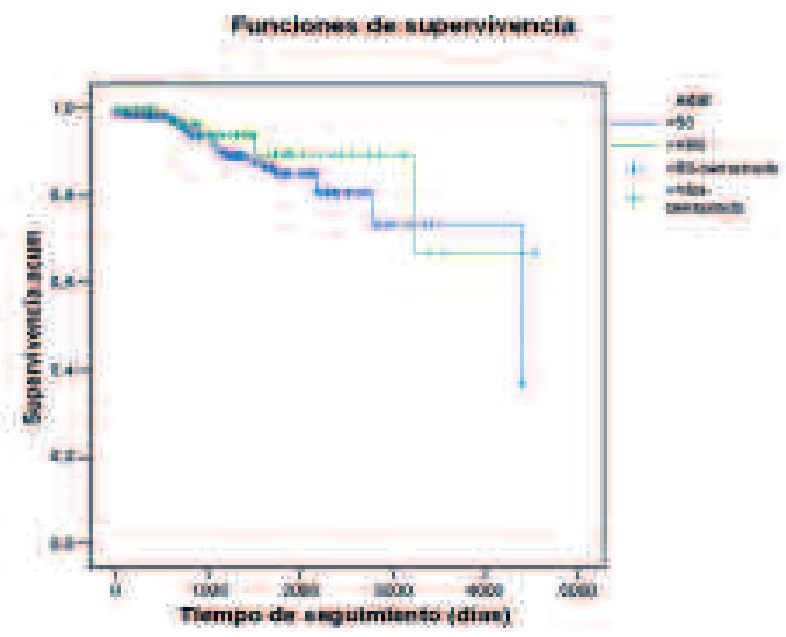

Fig. 3: Curva de supervivencia según la edad de menos de 50 años y de 50 o más años $(p=0,323)$. 
tes $<50$ años a los 2, 5 y 10 años de seguimiento es del $91,41 \%, 81,93 \%$ y $61,45 \%$ respectivamente. $\mathrm{Si}$ analizamos los pacientes $\geq 50$ años a los 2,5 y 10 años de seguimiento apreciamos unas tasas de supervivencias del $90,86 \%, 73,18 \%$ y del $58,28 \%$ respectivamente.

Pero cuando consideramos el factor sexo, entre aquellos pacientes de menos de 50 años no parece existir diferencias significativas en la supervivencia de los mismos, pero cuando analizamos aquellos pacientes de 50 o más años entonces encontramos una mayor supervivencia entre los hombres respecto a las mujeres (Log-rank; p=0,038) (figs. 4 y 5).

\section{DISCUSIÓN}

La distribución por edades del melanoma de úvea es un tema que nos preocupaba en la práctica clínica diaria ya que teníamos la impresión de diagnosticar y tratar a una población afecta de melanoma uveal más joven de lo publicado, así pues decidimos revisar nuestra base de datos y compararlos con la literatura.

Algunas series muestran que la media de edad de presentación del melanoma de úvea está en torno a los 55 años $(1,4,5)$. En otras la media de presentación es algo más tardía, hacia los 60 años (6-9). La etnia juega un factor importante en cuanto a la edad de presentación, así se describe que en pacientes asiáticos (japoneses) es menor que en pacientes caucásicos (55,2 años), en pacientes chinos e hispanos también es inferior (52,4 años) y en pacientes indios incluso bastante menor (45,7 DE 14,2 años) (10-12). En nuestra serie de pacientes encontramos una media de edad de 60,09 DE 1,67 años comparable con la de las series publicadas en pacientes caucásicos (todos nuestros pacientes lo son) (6-9). Se postula que el grado de pigmentación cutánea podría ser un factor relativo de protección para el desarrollo de este tipo de tumoraciones, al igual que ocurre con el melanoma cutáneo, pero se desconoce porque en las razas asiáticas o en los hispanos los melanomas se presentan a una edad más precoz (12).

Cuando analizamos a los pacientes de menos de 20 años hemos encontrado un porcentaje de prevalencia sobre el total de los melanomas de $0,2 \%$, muy similar al de otras publicaciones que oscila entre el 0,6 al 1,6\% (5), pero en algunas series se ha encontrado una prevalencia del $11,4 \%$ al $19 \%$ en pacientes hispanos y asiáticos entre 19 y 30 años (4). También se analizaron aquellos pacientes diagnosticados de un melanoma coroideo antes de $30 \mathrm{y}$ 40 años, y se encontraron $6(2,0 \%)$ y $38(12,5 \%)$ casos respectivamente. Estos porcentajes de pacientes jóvenes con el diagnóstico de melanoma de úvea, aunque se ajustan a lo descrito en otras series, no dejan de ser relativamente elevados, por lo que no debemos descartar el diagnóstico de esta tumoración por la edad del paciente.

En nuestro estudio no hemos evidenciado diferencias estadísticamente significativas entre la localización anatómica de los tumores (iris, coroides y cuerpo ciliar) y la edad de los pacientes en el momento del diagnóstico. Así como tampoco
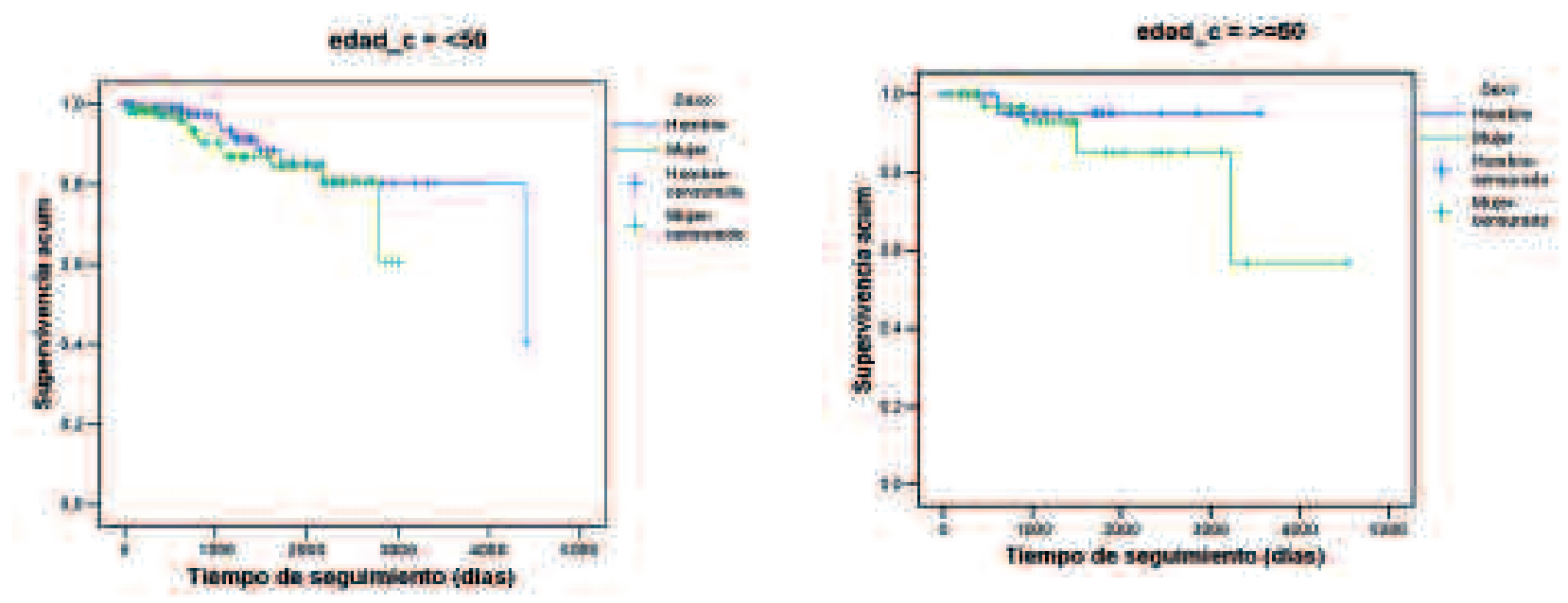

Fig. 4 y 5: Curvas de supervivencia diferenciando el sexo entre pacientes de menos de 50 años y de 50 o más años. 
hemos podido encontrar relaciones entre el tamaño tumoral y la edad de presentación.

Cuando analizamos la edad de los pacientes (menos de 20, 30, 40 ó 50 años respecto a los de más de 20, 30, 40 ó 50) en relación a su estado sistémico, no hemos encontrado diferencias estadísticamente significativas entre estos diferentes grupos. Por lo tanto no hemos podido demostrar un pronóstico vital diferente según la edad de presentación de los pacientes. Cuando consideramos el factor sexo, hemos evidenciado entre aquellos pacientes de 50 o más años, una mayor supervivencia entre los hombres respecto a las mujeres (Log-rank; $p=0,038$ ).

Clásicamente se ha descrito que la edad más joven en el momento del diagnóstico se correlaciona con una mayor tasa de supervivencia (10). En nuestros pacientes jóvenes $<50$ años hemos encontrado supervivencias a los 2, 5 y 10 años de seguimiento del $91,41 \%, 81,93 \%$ y $61,45 \%$ respectivamente, y en nuestros pacientes $\geq 50$ años estas cifras son del $90,86 \%, 73,18 \%$ y del $58,28 \%$ respectivamente, en todos los casos sin tener en cuenta el tamaño tumoral ni el tratamiento utilizado. Kujala describe tasas de supervivencia a los 5 años del $69 \%$, a los 15 años del $55 \%$ y del $51 \%$ a los 25 años (9), Seddon describe tasas a los 5, 10 y 15 años de $74 \%, 63 \%$ y $55 \%$ respectivamente (13), y Kroll tasas a los 5 y 10 años de $75,6 \%$ y $62,3 \%$ de supervivencia (14), similares a muchas otras series.

La mejor supervivencia en los pacientes jóvenes se atribuye a que acuden antes al especialista para ser diagnosticados con mayor rapidez que los pacientes más mayores, presentando de esta manera tumores de mejores características en el momento del diagnóstico $(3,5)$.

Podemos concluir que el melanoma de úvea en nuestro medio sigue un patrón de distribución por edades similar al descrito en otras series de otros países, no siendo infrecuente el diagnóstico en pacientes menores de 40 años. No hemos podido encontrar diferencias significativas entre la edad de los pacientes y el pronóstico vital, aunque cuando hemos analizado subgrupos, los hombres de más de 50 años parece que presentan una mejor supervivencia.

De todas maneras es importante aumentar el número de pacientes estudiados así como el segui- miento de los mismos para establecer resultados más concluyentes.

\section{BIBLIOGRAFÍA}

1. Egan KM, Seddon JM, Glynn RJ, Gragoudas ES, Abert DM. Epidemiologic aspects of uveal melanoma. Surv Ophthalmol 1988; 32: 239-251.

2. Bergman L, Seregard S, Nilsson B, Ringborg U, Lundell $G$, Ragnarson-Olding B. Incidence of uveal melanoma in Sweden from 1960 to 1998. Invest Ophthalmol Vis Sci 2002; 43: 2579-2583.

3. Singh AD, Shieds CL, Shields JA, Sato T. Uveal melanoma in young patients. Arch Ophthalmol 2000; 118: 918-923.

4. Báez-González DE, Martínez Jardon CS, Rodríguez-Reyes AA, Salcedo-Casillas G. Características clínico-patológicas del melanoma uveal en pacientes mexicanos menores de 30 años de edad. Arch Soc Esp Oftalmol 2004; 79: $543-$ 548 .

5. Shields CL, Shields JA, Milite J, De Potter P, Sabbagh R, Menduke H. Uveal melanoma in teenagers and children. A report of 40 cases. Ophthalmology 1991; 98: 16621666.

6. Isager P, Ehlers N, Overgaard J. Prognostic factors for survival after enucleation for choroidal and ciliary body melanomas. Acta Ophthalmol Scand 2004; 82: 517-525.

7. Richtig E, Langmann G, Mullner K, Smolle J. Ocular melanoma: epidemiology, clinical presentation and relationship with dysplastic nevi. Ophthalmologica 2004; 218 : 111-114.

8. Kaiserman I, Anteby I, Chowers I, Blumenthal EZ, Kliers I, Pe'er J. Post-brachytherapy initial tumour regression rate correlates with metastasic spread in posterior uveal melanoma. Br J Ophthalmol 2004; 88: 892-895.

9. Kujala E, Makitie T, Kivela T. Very long-term prognosis of patients with malignant uveal melanoma. Invest Ophthalmol Vis Sci 2003; 44: 4651-4659.

10. Sakamoto T, Sakamoto M, Yoshikawa H, Hata Y, Ishibashi T, Ohnishi Y, et al. Histologic findings and prognosis of uveal malignant melanoma in japanese patients. Am J Ophthalmol 1996; 121: 276-283.

11. Biswas J, Kabra S, Krishnakumar S, Shanmugam MP. Clinical and histopathological characteristics of uveal melanoma in Asian Indians. A study of 103 patients. Indian J Ophthalmol 2004; 52: 41-44.

12. Hudson HL, Valluri S, Rao NA. Choroidal melanomas in Hispanic patients. Am J Ophthalmol 1994; 118: 57-62.

13. Seddon JM, Albert DM, Lavin PT, Robinson N. A prognostic factor study of disease-free interval and survival following enucleation for uveal melanoma. Arch Ophthalmol 1983; 101: 1894-1899.

14. Kroll S, Char DH, Quivey J, Castro J. A comparison of cause-specific melanoma mortality and all-cause mortality in survival analyses after radiation treatment for uveal melanoma. Ophthalmology 1998; 105: 2035-2045. 